\title{
Flexibilización laboral en el sector de la salud: aproximación desde la gestión de la seguridad y la salud en el trabajo*
}

\section{Labor Flexibility in the health sector: an approach from the management of safety and health at work}

Yuber Liliana Rodrígue: Rojas**

Recibido: 11 de febrero de 2014

Revisado: 13 de abril de 2014

Aceptado: 27 de junio de 2014

Universidad Agustiniana

Jorge Hernando Molano Velandia ****

Universidad Nacional de Colombia

\section{RESUMEN}

El presente artículo busca describir los efectos y exigencias de la flexibilidad laboral, especialmente en el sector de la salud, y las implicaciones que tiene para la gestión de la salud y la seguridad laboral. Para lograr este objetivo, se realiza una revisión temática que evidencia que la salud es uno de los sectores más impactados por dicho fenómeno. Lo anterior se ve reflejado en la inestabilidad laboral de los trabajadores, la cual es generada por el cambio en las modalidades de contratación; la susceptibilidad de despido y de rotación; en el descentramiento del trabajo; la segmentación de la mano de obra y la reducción de la libertad sindical. Al ser la gestión de la salud y seguridad en el trabajo un proceso sistemático y organizado, orientado a la protección de la salud y al fomento de la seguridad de la población laboral, permite la incorporación de

\footnotetext{
* Artículo de investigación.

** Docente de posgrados Universitaria Agustiniana. Mg. en Salud y Seguridad en el Trabajo - perfil investigativo. Correo electrónico: ylrodriguezr@gmail.com, yuber.rojas@uniagustiniana.edu.co Autor de correspondencia.

*** Doctor en ciencias económicas. Director Nacional de Extensión, Innovación y Propiedad Intelectual de la Universidad Nacional de Colombia. Líder de la línea de investigación en Organización, Gestión y Regulación de la Salud y Seguridad en el Trabajo de la Maestría en Salud y Seguridad en el Trabajo de la Universidad Nacional de Colombia. Correo electrónico: jhmolanov@unal.edu.co
} 
componentes que llevan a complementar la gestión de las organizaciones en aspectos que no han sido tradicionalmente tenidos en cuenta en un nivel suficiente para lograr beneficios organizacionales y de las personas.

Palabras clave: trabajo, salud, gestión en salud, organización y administración, recursos humanos.

\section{ABSTRACT}

This article aims to describe the effects and demands of labor flexibility, especially in the health sector, and the implications for the management of health and safety at work. To this end, we did a literature review, which evidences that the health sector is one of the most affected by this phenomenon. This is reflected in the instability of workers, generated by the changes made within recruitment processes; the susceptibility of dismissal and rotation; decentering at work; segmentation of labor and reduction of freedom of association. As the management of health and safety at work is a systematic and organized process aimed at protecting health and promoting the safety of the workforce, it allows the incorporation of components leading to complement the management of organizations in aspects that have not traditionally been considered in sufficient levels to achieve personal and organizational benefits.

Keywords: Work, health, health management, organization and management, human resources.

\section{INTRODUCCIÓN}

La globalización es la actual fase del desarrollo del capitalismo, considerada como la aceleración de la tendencia por parte de las empresas y de los países, que establece una red mundial para el intercambio y posicionamiento en el mercado mundial. Ahora bien, Coriat define la globalización "como una nueva fase de la internacionalización de los mercados, que pone en dependencia recíproca a las empresas y a los países, en grados absolutamente originales e inigualados en el pasado". Desde este punto de vista, se puede decir que el capitalismo es la fase de mayor internacionalización y desverticalización de las empresas, la cual busca poner fin a la hegemonía del modelo de organización de empresas jerárquicas y rígidas, con la cual se expandían mundialmente las empresas por medio de la producción masiva, así como las economías de escala y las mercaderías estandarizadas de bajo costo ${ }^{2}$.

La desverticalización se ha traducido en flexibilización laboral. El presente artículo se basa en dicho fenómeno, no sólo desde el punto de vista conceptual, sino en el análisis de las discrepancias de la población laboral en sus condiciones laborales, especialmente en el sector salud. Así mismo, el presente artículo busca analizar las implicaciones que dicho factor tiene para la gestión de salud y seguridad en el trabajo.

El nuevo modelo de competitividad exige a las empresas flexibilizar los parámetros de producción. En respuesta a ello, han implementando estrategias empresariales con la intención de interrelacionar acciones que por sí solas no pueden desarrollar, acudiendo a las alianzas y a la subcontratación de servicios. Las empresas de servicios son parte de esta estrategia, a ellas les han atribuido funciones que antes eran desarrolladas por las compañías. Esta situación ha generado

1 CORIAT, Benjamín Globalización de la economía y dimensiones macroeconómicas de la competitividad. CONICIT y el CREEDLA del CNRS.1994. 12 p.

2 AÑEZ, Carmen. Estrategias de contratación de recurso humano en las empresas de servicios. Fermentum [Internet]. 2007 [citado 13 ago 2011]; 48: 123-141. Disponible en: http://www2.scielo.org.ve/ pdf/ferm/v17n48/art07.pdf. 
la estructuración de nuevas relaciones laborales en el mundo del trabajo, que son el resultado de un nuevo régimen de acumulación. Este último se fundamenta en los supuestos de la competitividad internacional, el mejoramiento de la productividad y la reducción de costos, cuyo fin busca generar una nueva dinámica del capital que permita ampliar los mercados para generar más empleos3. Este tipo de cambios también se han presentado en la gestión de la salud y la seguridad en el trabajo. Los resultados reflejan que estas entidades están siendo utilizadas por las organizaciones más grandes para insertarse en el mercado global, a la vez que buscan reducir costos laborales4.

En este sentido, los procesos de flexibilización en materia de derechos de trabajo en Colombia, y en otros países, corresponden a relaciones de índole económica, política y social que sustentan una amplia gama de cambios estructurales, políticos y normativos en el ámbito laboral para afrontar los retos que impone una economía globals.

La flexibilización implica necesariamente la modificación de la normativa jurídica, de las estructuras estatales, de los modelos y políticas macroeconómicas y microeconómicas, así como la modernización y acceso a la tecnología, con el fin de adaptar los modelos productivos a las nuevas tendencias de globalización e integración comercial. Así mismo, para garantizar un crecimiento económico sostenible que permita cumplir el objetivo fundamental de la economía: ofrecer

3 MILANO, Elías. Del Fordismo a la flexibilidad laboral: supuestos, crisis, y realidades de la regulación social. Caracas. Biblioteca Omegalfa.1997. p.24

4 Ibíd. p. 34

5 ARENAS, Eraclio, PIEDRAHITA, Camilo y PLATA Juan Miguel. Marco jurídico de las cooperativas de trabajo asociado. Opinión Jurídica [Internet]. 2007 [citado 13 ago 2011]; 6 (11). p. 34-45. Disponible en: http://redalyc.uaemex.mx/src/inicio/ArtPdfRed. jsp?iCve=94501103. el mayor bienestar posible al ser humano a través de la maximización de unos recursos escasos ${ }^{6}$.

La problemática socio-económica, producto del desempleo y el subempleo, que no ha podido ser respondido exitosamente por parte de muchos Estados, ha planteado la necesidad de crear nuevas formas y modalidades de trabajo que permitan generar recursos en el sector real de la economía nacional, así como posibilitar la movilidad de las líneas de consumo y producción. Esta posición se encuentra fundamentada en la concepción neoclásica de la economía que señala como responsable de los altos niveles de desempleo a la rigidez del mercado y contratación laboral, la cual se refleja en la normativa jurídica que regula dichas materias y que es producto de la intervención estatal en la economía?.

Este contexto ha influido en la flexibilización de la contratación laboral actual, la cual se ha orientado hacia esquemas no tradicionales, tales como: la tercerización (outsourcing), la contratación o subcontratación de servicios mediante orden de servicios, 0 a través de empresas de servicio temporales y de cooperativas de trabajo asociado. Es curioso que estas últimas empresas se triplicaron en Colombia entre los años 2002 y 2007, y el número de trabajadores comprometidos aumentó a una tasa anual promedio del $41 \%$, una cifra muy superior a la tasa de crecimiento del empleo nacional, que fue inferior al $2 \%$ anual en el mismo periodo 8,9 .

6 ARENAS, Eraclio, PIEDRAHITA, Camilo y PLATA, Juan Miguel. Flexibilización y derecho laboral. Opinión Jurídica [Internet]. 2007 [Citado 13 ago 2011]; 5 (9): 163-171. Disponible en: http://redalyc. uaemex.mx/pdf/945/94550909.pdf.

7 Ibíd.

8 FARNÉ, Stefano. Las cooperativas de trabajo asociado en Colombia: Balance de la política gubernamental, 2002-2007. Economía Institucional [Internet]. 2008 [citado 13 ago 2011]; 10 (18): 261-285. Disponible en: http://www.economiainstitucional.com/pdf/No18/ sfarne18.pdf.

9 RODRÍGUEZ, Yuber Liliana y MOLANO, Jorge. Gestión de la seguridad y salud en el trabajo frente a las formas de vinculación en 
Por otro lado, con respecto al sector de la salud, Robinson señala en el análisis que hace de las relaciones entre hospitales y médicos el modo como la Teoría de los Recursos y las Capacidades puede ser usada para explicar la tendencia a la integración vertical entre estos dos eslabones de la cadena de prestación de los servicios de la salud. El marco de referencia que construye este autor desde la teoría económica tiene en cuenta los aportes de la economía de los costos de transacción, la teoría de la agencia, así como la teoría de los recursos y las capacidades ${ }^{10}$.

En el sector de la salud se han realizado algunas aplicaciones de integración vertical en organizaciones. Se sugiere que la integración vertical en el sector debería ser una estrategia acertada, lo cual se intentó verificar empíricamente en Estados Unidos. En este sentido, se encontró que los hospitales aumentaban sus ingresos cuando se integraban verticalmente con los proveedores de los servicios médicos, de enfermería, de fisioterapia, entre otros. Los resultados parecieron ser convincentes, y en términos estadísticos fueron significativos ${ }^{11}$.

Hoy en día es común encontrar dentro del sector de la salud que los trabajadores se contraten a través

un consorcio empresarial. Universidad Nacional de Colombia. En: La investigación en Salud y Seguridad en el trabajo Realidades y desafíos actuales. Bogotá. D.C: Universidad Nacional de Colombia. 2014. 189 p.

10 TORRES, Sergio y ANGEL, Víctor Manuel. Outsourcing de servicios en la prestación de servicios de salud de Bogotá. Gerencia y Políticas de Salud [Internet]. 2004. [citado 13 ago 2011]; No. 3 (7). P. 101 - 113. Disponible en: http://redalyc.uaemex.mx/src/inicio/ ArtPdfRed.jsp?iCve=54500707.

11 CODY, Marisue. Vertical integration strategies: Revenue effects in hospital and medicare markets. Hospital \& Health Services Administration. University of Arkansas for Medical Sciences, College of Nursing [Internet]. 1996 [Citado 13 ago 2011]; 41 (3): 343-357. Disponible en: http://www.ncbi.nlm.nih.gov/pubmed?term=Vertical\%20integration $\% 20$ strategies $\% 3 \mathrm{~A} \% 20$ revenue $\% 20$ effects\%20in\%20hospital $\% 20$ and\%20Medicare\%20markets. de cooperativas de trabajo o a través de órdenes de prestación de servicios. No obstante, dichas modalidades generan "inestabilidad laboral", por esta razón, para incrementar sus ingresos, los profesionales de la salud tienden a buscar múltiples empleos, así como a someterse al trabajo por turnos. A pesar de ello, los trabajadores son evaluados a través del cumplimiento de metas e indicadores, lo que a su vez genera efectos negativos sobre sus condiciones de salud, su bienestar y su calidad de vida.

Al modificarse las relaciones laborales entre el contratante y el trabajador, se transforman las actividades propias de los procesos y procedimientos de la salud y la seguridad en el trabajo dentro de la organización; lo que a su vez puede afectar las condiciones de salud y de bienestar de los trabajadores que laboran en determinada organización o sector, tal es el caso de los hospitales.

La flexibilización laboral ha sido descrita por diversos autores, uno de ellos es Echeverría quien afirma que corresponde a un “... proceso de generación de condiciones económicas, legales y culturales que permitan al conjunto del sistema productivo, las empresas y los trabajadores adaptarse al escenario de competencia comercial que surge del proceso de globalización de la economía y del desarrollo de las nuevas tecnologías, especialmente de la informática y en las comunicaciones" ${ }^{12}$.

Por otra parte, Rincón y Rodríguez indican que la flexibilización puede considerarse como "una estrategia vinculada a la organización del trabajo y a las

12 DEPESEX/BCN/Serie Estudios. La flexibilidad laboral en los países de economías avanzadas y de América Latina. El caso chileno. Biblioteca del Congreso Nacional de Chile [Internet]. 2005 [citado 13 ago 2011]; 318. Disponible en: http://www.bcn. cl/bibliodigital/pbcn/estudios/2005/estudios_pdf_estudios/ nro318.pdf. p.3. 
relaciones laborales" ${ }^{13}$. Esta idea se complementa con lo señalado por Coller, para quien quien la flexibilización es el "conjunto de prácticas empresariales que se ponen en marcha para adaptar la organización a un entorno cambiante" ${ }^{14}$.

En este sentido, se puede afirmar que la flexibilización laboral también ha sido producto de cambios tecnológicos, y ha surgido como respuesta a los fenómenos de la organización del trabajo ${ }^{15} 16$. La flexibilización laboral trae consigo efectos sobre las condiciones contractuales de los trabajadores, al generar modificaciones en su relación laboral con las organizaciones en las cuales se desempeñan ${ }^{17}$.

Se han identificado dos tipos de flexibilización: la interna y la externa. La primera corresponde a las prácticas laborales y de organización del trabajo que se dan dentro de las empresas. Esta se relaciona con cuatro elementos: las tareas, la organización variable de las horas, la disponibilidad del lugar de trabajo y la organización versátil durante el ciclo vital del trabajador. La flexibilización externa corresponde a las prácticas constantes de reducción e incremento del personal, las cuales varían de acuerdo a las necesidades de la empresa. Esta se presenta a través de empresas de servicios

13 RINCÓN, María T. y RODRÍGUEZ, Isabel Flexibilización Laboral en el sector salud: Una estrategia en la Gobernación del Zulia. Gaceta Laboral [Internet]. 2005 [citado 13 ago 2011]; 11 (3). Disponible en: http://www.scielo.org.ve/scielo.php?pid=S131585972005000300003\&script=sci_arttext.pP.3

14 COLLER, Xavier La empresa flexible: Estudio sociológico del impacto de la flexibilidad en el proceso de trabajo. Centro de Investigaciones Sociológicas. Madrid, España; 1997. p. 45.

15 DEPESEX/BCN/Serie Estudios. Op cit.

16 ECHEVERRÍA, Magdalena. Aportes para el debate sobre flexibilidad laboral. Dirección del Trabajo. Colección IDEAS. 2003.

17 RODRÍGUEZ, Yuber Liliana y MOLANO, Jorge. Adaptación de una herramienta para la evaluación de la gestión de la salud y seguridad en el trabajo. En: El Hombre y la Máquina. 2012. vol. 40. p. 7 -21. temporales, staff, contrapartidas estratégicas, outsourcing y equipos interempresas ${ }^{18,19,20}$.

Otros autores como Lizama refieren que "la flexibilidad laboral ha admitido diversas categorías, siendo las más importantes la fórmula de la flexibilidad de costos salariales, la flexibilidad numérica y la flexibilidad funcional" ${ }^{21}$.

La flexibilidad de costos salariales guarda relación con los mecanismos que otorga la Ley para que el empleador pueda reducir costos de producción, a través de la reducción en la remuneración y otras prestaciones laborales. Así, por ejemplo, se han creado distintos contratos de trabajo que no responden al contrato tradicional y que, por regla general, se han dirigido a atraer mano de obra joven durante cortos períodos de tiempo. La flexibilidad numérica hace referencia a la posibilidad legal de reducir la cantidad de trabajadores en el proceso productivo, la cual puede darse mediante la reducción de la jornada de trabajo o a través de la reducción del número de trabajadores en ciertos periodos del año. Finalmente, la flexibilidad funcional se refiere a la disminución de los costos en el proceso productivo, bien sea a través de cambios en la forma de organizar la empresa en lo relativo a la duración de la jornada de trabajo, la polifuncionalidad, la suspensión del vínculo laboral o mediante el despido $0^{22}$.

Adicionalmente, existen dos tipos de clasificación diferentes de la flexibilización laboral: la cualitativa y la

18 DEPESEX/BCN/Serie Estudios. Op cit.

19 ECHEVERRÍA. Op cit.

$20 \mathrm{KLAU}$, Friedrich y MITTELSTADT, Axe. Labour market flexibility. OECD [Internet]. Disponible en internet: http://www.oecd.org/ dataoecd/55/25/35558438.pdf.

21 LIZAMA, Claudio. Flexibilidad laboral en la Unión Europea y Estados Unidos. Revista de la Escuela de Derecho de la Universidad de Chile. 2003. p. 35-51.

22 Ibíd. 
cuantitativa. La cualitativa se relaciona con la capacidad de adaptación del trabajador en múltiples puestos de trabajo y a la posibilidad de movilidad interna dentro de la compañía. La cuantitativa concierne al incremento o reducción del personal contratado ${ }^{23 .}$

Lo anterior se relaciona con lo planteado por Ochoa y Gamboa, para quienes la flexibilización en la gestión del recurso humano incluye la reducción del personal de planta, así como la incorporación de funcionarios contratados bajo modalidades que facilitan el despido y la movilidad del personal (intraorganizacionales e interorganizacionales), lo que conduce a una inestabilidad laboral ${ }^{24}$. Adicionalmente, se promueve la fragmentación de la fuerza de trabajo y se impone una nueva forma de organización de la producción que conlleva a contrataciones cortas y ocasionales y a acceder a contratos que dependen de las fluctuaciones en la demanda de los bienes producidos o de los servicios prestados ${ }^{25}$.

Tal como describió Ermira en 1999, se habla entonces del paradigma de las relaciones laborales postmodernas, que obedecen a las siguientes características: descentramiento del trabajo, segmentación de la mano de obra, inestabilidad en el empleo, individualización de la relación laboral y, como resultado, se produce el abaratamiento del costo de la mano de obra ${ }^{26}$. Lo anterior reduce el salario real y se refuerza la flexibilización de los horarios en función de las necesidades productivas,

23 ECHEVERRÍA. Op cit.

24 OCHOA, Haydée y GAMBOA, Teresa. Hacia la Gerencia Pública en Venezuela: el caso de la administración tributaria. En: Revista Centroamericana de Administración Pública. 1999, № 37. p. 93-116.

25 LUCENA, Héctor. Relaciones de Trabajo en el Nuevo Siglo. Fondo editorial Tropykos. 2004.

26 ERMIDA, Oscar. Globalización y relaciones laborales. En: Revista Venezolana de Gerencia [Internet]. 1999 [citado 13 ago 2011], No. 4 (9). Disponible en: http://www.cinterfor.org.uy/public/spanish/ region/ampro/cinterfor/publ/sala/ermida/globaliz/globaliz.pdf. tal como se muestra en: la prolongación de las jornadas de trabajo, la modificación en los periodos de descanso semanal y las vacaciones de los trabajadores, así como en el trabajo por turnos rotativos. Además, se reduce la libertad sindical y el derecho a crear asociaciones de carácter social y cooperativo ${ }^{27}$.

\section{METODOLOGÍA}

Este artículo describe los efectos y exigencias de la flexibilidad laboral, especialmente en el sector de la salud, así como las implicaciones existentes dentro de la gestión de la salud y la seguridad en el trabajo. Para llevar a cabo la investigación se realizó una revisión temática, la cual muestra que la salud es uno de los sectores más impactados por dicho fenómeno.

Esta revisión temática permitió identificar diferentes textos a partir del método iterativo incremental. Dicha información fue analizada a partir de la identificación de las tendencias en la literatura consultada. Se consideraron como categorías centrales del análisis el concepto de gestión de la seguridad y salud en el trabajo, los efectos y las exigencias de la flexibilización laboral sobre el trabajador y sobre la organización.

\section{RESULTADOS}

\section{La flexibilización laboral en el sector de la salud y sus efectos}

Si bien las transformaciones laborales se han visto en todos los sectores, estas se evidencian específicamente en el sector de la salud, por lo que estas modificaciones deben comprenderse en el marco de los procesos de

27 RINCÓN y RODRÍGUEZ. Op cit. 
reestructuración de las organizaciones del sector, de las manifestaciones particulares y de la globalización del sistema correspondiente.

Considerando que el sector de la salud es uno de los que más hace uso de los recursos humanos, un estudio que mida la calidad y el funcionamiento de sus sistemas de salud necesita enfocarse en el potenciamiento de dicho recurso y en el mejoramiento de las características de su aplicación para que, a su vez, se logre mejorar significativamente su desempeño. Sin embargo, el fenómeno que se observa en la actualidad señala lo contrario debido a los efectos que ha causado la flexibilización en el sector, tales como: reducción de empleos de planta y contratación de los trabajadores según las necesidades detectadas por cada institución o por la demanda misma de los servicios, lo que se relaciona con la llamada reestructuración productiva ${ }^{28}$.

En este contexto, como bien lo señala Ermida, la contratación temporal produce la individualización de la vinculación legal de la fuerza de trabajo en detrimento de las organizaciones de los trabajadores ${ }^{29}$. De este modo, la flexibilización no ha sido ajena a la problemática general de las relaciones laborales que se observa en la prestación de servicios de la salud. Adicionalmente, los cambios en las relaciones contractuales están afectando de manera negativa la organización de los trabajadores de la salud, como lo son las agremiaciones sindicales. Por otro lado, los incentivos laborales corresponden únicamente a la posibilidad de acceder a algunas capacitaciones y no a otro tipo de beneficios, en comparación con la existencia del contrato estable de trabajo. A partir de entrevistas realizadas al personal de salud se evidencia que la situación de incertidumbre, ocasionada por el temor hacia el

28 MOTA, Ana Elizabete Cultura da Crise e Seguridade Social: Um Estudo Sobre as Tendências da Previdência e da Assistência Social Brasileira nos Anos 80 e 90. São Paulo: Cortez, 1995. p.6

29 ERMIDA, Oscar Op cit. desempleo y a la falta de estabilidad laboral, es el elemento más destacado del empleo temporal ${ }^{30}$.

La Ley 50 de 1990 generó diversos efectos en Colombia, tales como: formalización del empleo temporal; autorización de la jornada de seis horas por turnos para evitar el pago de horas extras y compensatorios; posibilidad de firmar contratos a término fijo inferiores a un año; introducción de contratos civiles de prestación de servicios para suprimir el pago de prestaciones sociales; eliminación de la obligación de reintegro para trabajadores con más de diez años de servicio; permiso de despidos colectivos cuando el empleador lo estime necesario por adecuación tecnológica o razones económicas; supresión de la retroactividad de las cesantías para aquellos que se acojan al sistema privado de los fondos de pensiones y cesantías y para los que ingresen a laborar a partir de la vigencia de la ley y, además, implantación del salario integral ${ }^{31}$.

Posteriormente, la Ley 789 del 27 de 2002 ratifica la flexibilización en el sector, y señala elementos que permiten responder a las formas de contrato que buscan ajustarse a las nuevas formas de trabajo, de organización y de jornada laboral32. En el Cuadro 1 se aprecian los efectos que ha generado la flexibilización laboral en el sector salud.

30 OBSERVATORIO DE RECURSOS HUMANOS EN SALUD. Relaciones laborales en el sector salud. El Sistema General de Seguridad Social en Salud de Colombia: El caso de la ciudad de Cali. Ministerio de la Protección Social y Organización Panamericana de la Salud. 2006. 86 p.

31 COLOMBIA. CONGRESO DE LA REPÚBLICA. Ley 50 (28, diciembre, 1990). Por la cual se introducen reformas al Código Sustantivo del Trabajo y se dictan otras disposiciones. Bogotá D.C.: Alcaldía de Bogotá.

32 COLOMBIA. CONGRESO DE LA REPÚBLICA. Ley 789 (27, diciembre, 2002). Por la cual se dictan normas para apoyar el empleo y ampliar la protección social y se modifican algunos artículos del Código Sustantivo de Trabajo. 
Yuber Liliana Rodríguez Rojas, Jorge Hernando Molano Velandia

Cuadro 1. Efectos de la flexibilización en el sector salud

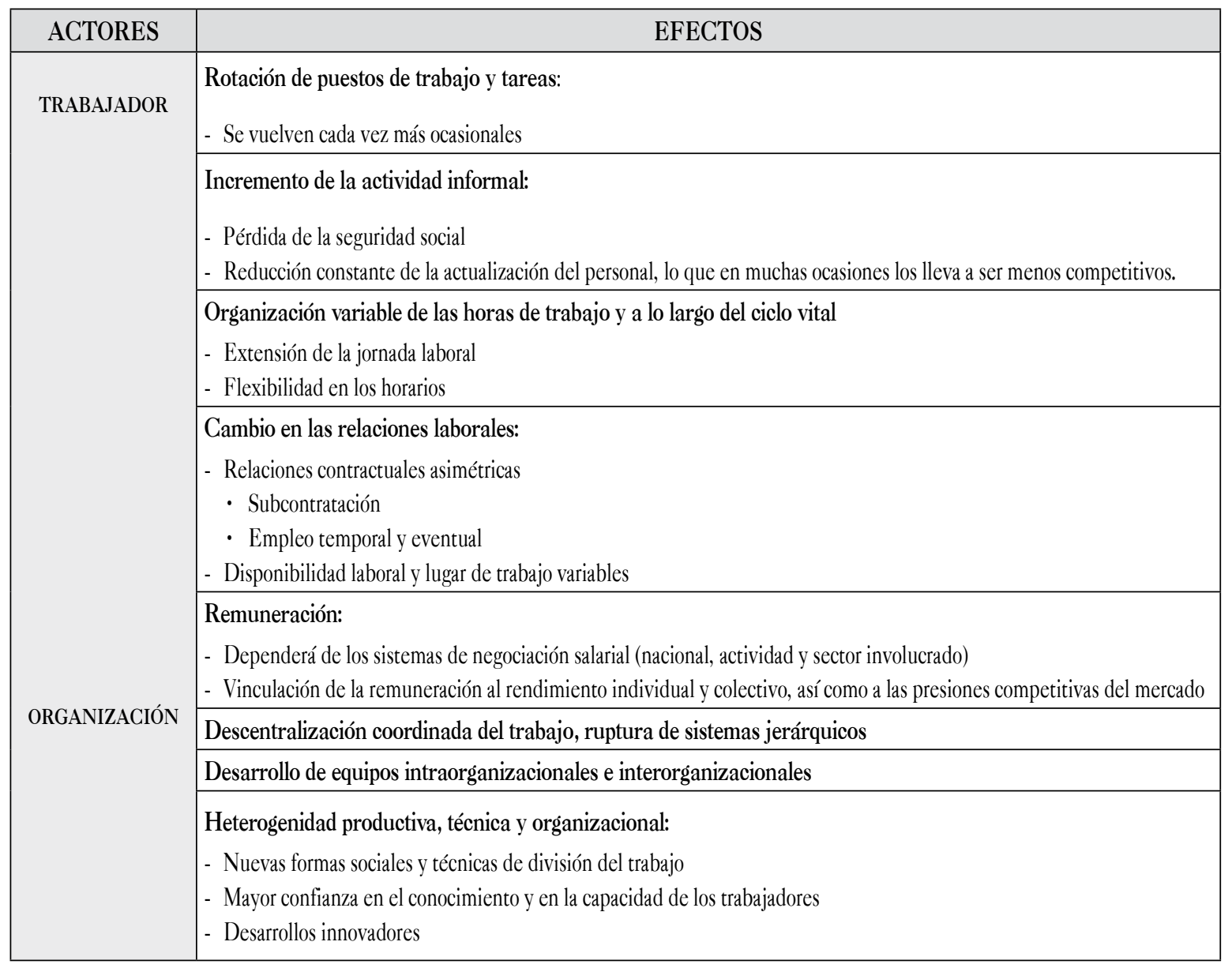

Fuente: elaboración propia basada en Rincón M y Rodríguez I, 2005; Torres S y Angel V, 2004; Echeverría M, 2003.

\section{La salud y seguridad en el trabajo y su gestión}

Los cambios en las estructuras empresariales, producto de la flexibilización laboral, han fragmentado los ciclos productivos y, por consiguiente, han favorecido la formación de redes de subcontratación en pequeñas y medianas empresas. Lo anterior corresponde a la estructura típica de la economía globalizada. Sin embargo, esta estructura cuenta con formas organizacionales tradicionales y actuales ${ }^{33}$ que impiden que algunos

33 VALDÉS, Fernando. Descentralización productiva y desorganización del derecho del trabajo. Universidad Complutense de Madrid. [citado 13 nov 2011]. Disponible en: http://www.ruct.uva.es/pdf/ Revista\%202/202.pdf procesos dentro de las instituciones no cumplan con las necesidades generadas a partir de los cambios producidos por dicha flexibilización, tal es el caso del sector de la salud y la seguridad de los trabajadores.

Con el ánimo de reducir los efectos anteriormente señalados, es necesario abordar este tema desde diferentes áreas del conocimiento, principalmente para reducir aquellos efectos que afectan directamente al trabajador, pues de alguna manera las organizaciones han logrado identificar y proponer estrategias para responder activamente a los cambios que ha traído la globalización.

En este sentido, se debe acudir a lo que en la actualidad se comprende como salud y seguridad en el trabajo 
(SST), la cual, según Betancourt, consta de tres ámbitos o categorías que corresponden a las condiciones de trabajo, las condiciones de salud y las condiciones de vida. A su vez, dichas condiciones laborales engloban cuatro elementos fundamentales que se relacionan con el medio ambiente, la tarea, la organización del trabajo y la salud del trabajador ${ }^{34}$.

En contraste, las condiciones de salud-enfermedad se encuentran representadas por los accidentes y las enfermedades del trabajo, las manifestaciones tempranas o sub-clínicas y las manifestaciones saludables. Las condiciones de vida se pueden localizar en los mediadores singulares, particulares y generales. Además de lo señalado, y en estrecha relación con las condiciones de trabajo, se deben añadir las medidas de higiene y seguridad que existan en el centro de trabajo ${ }^{35}$.

Ahora bien, para poder garantizar la salud de los trabajadores y el mantenimiento de la productividad de las organizaciones, se requiere estudiar integralmente el proceso de trabajo y su relación con la salud, apoyándose en diferentes áreas del conocimiento, siendo una de ellas la gestión.

La gestión es entendida como las “... actividades coordinadas para dirigir y controlar una organización (conjunto de personas e instalaciones con una disposición de responsabilidades, autoridades y relaciones)", mientras que un sistema de gestión es comprendido como un "... conjunto de elementos mutuamente relacionados o que interactúan para establecer la política y los objetivos y para lograr dichos objetivos" ${ }^{36}$. López, por su parte, describe la gestión como la ordenación metódica de actividades interdependientes y

34 BETANCOURT, Oscar. Enfoque alternativo de la salud y seguridad en el trabajo. IESS, Prevención es desarrollo. Quito 2007. 23 p..

35 Ibíd.

36 ISO. Norma Internacional ISO 9000:2005. Sistema de gestión de la calidad. Fundamentos y Vocabulario. 17 p. procedimientos relacionados que permiten la realización y buen hacer de una organización ${ }^{37}$.

En este orden de ideas, la Gestión de la Salud y Seguridad en el Trabajo (GSST) es entendida como un conjunto de procesos, procedimientos y actividades emprendidas por una o más personas, cuyo objetivo es integrar en las actividades de los otros la protección de la salud y fomentar la seguridad de la población laboral en una organización, contribuyendo con ello al desarrollo empresarial y a la sostenibilidad del negocio ${ }^{38}$.

Por su parte, para la Organización Internacional del Trabajo (OIT), la GSST es "un conjunto de herramientas lógico, caracterizado por su flexibilidad, que puede adaptarse al tamaño y la actividad de la organización, y centrarse en los peligros y riesgos generales o específicos asociados con dicha actividad. Su complejidad puede abarcar desde las necesidades básicas de una empresa pequeña que dirige el proceso de un único producto en el que los riesgos y peligros son fáciles de identificar, hasta industrias que entrañan peligros múltiples, como la minería, la energía nuclear, la manufactura química o la construcción”39. Además debe permitir su adaptación a los cambios en la actividad de la organización y a los requisitos legislativos ${ }^{40}$.

De acuerdo a la literatura no existe un sistema de GSST normalizado y reconocido en el mundo. Por esta razón cada organización se ve en la necesidad de crear

37 RUBIO, Juan Carlos Gestión de la prevención de riesgos laborales: OHSAS 18001 - Directrices OIT para su integración con calidad y medioambiente. Ediciones Díaz de Santos. España; 2006. 248 p.

38 Línea de investigación en gestión, organización y regulación de la salud y seguridad en el trabajo. Actas de reunión. Universidad Nacional de Colombia. 2010.

39 ORGANIZACIÓN INTERNACIONAL DEL TRABAJO. Sistema de gestión de la SST: una herramienta para la mejora continua. 1a. Ed. 2011. 4 p.

40 Ibíd. 
sus propios modelos o sistemas de gestión, teniendo en cuenta la complejidad de sus procesos de trabajo y sus necesidades ${ }^{41}$. Este fenómeno se debe a que los esfuerzos en la salud y la seguridad se han considerado acciones aisladas y se han centrado en aspectos técnicos, dejando de lado los aspectos organizativos y humanos ${ }^{42}$. Sin embargo, en los últimos años se han empezado a considerar factores organizacionales, culturales y de gestión que desempeñan un papel primordial en la generación de accidentes e incidentes ${ }^{43}$.

Así que algunos organismos como la OIT, se han puesto en la labor de crear un modelo de gestión orientador. Para esta organización los sistemas de GSST incluyen algunos elementos principales como son la política, la organización, la planificación y aplicación, la evaluación y la acción en pro de mejoras" ${ }^{4}$. No obstante, estos componentes deben ser analizados desde la perspectiva de la auditoría.

De acuerdo con lo descrito por Fernández, para que un sistema de GSST sea efectivo debe contener como mínimo los siguientes elementos ${ }^{45}$.

41 FERNÁNDEZ, Ricardo Los distintos sistemas de gestión de la salud y seguridad en el trabajo. Gestión Práctica de Riesgos Laborales. 2007; 44: 20.

42 FERNÁNDEZ, Beatriz, MONTES, José Manuel y VÁZQUEZ, Camilo. Desarrollo y validación de una escala de medición para el sistema de gestión de la seguridad laboral. Investigaciones Europeas de Dirección y Economía de la Empresa [Internet]. 2006 [citado 13 ago 2011]; 12 (3). p. 77-93. Disponible en: http://www.aedemvirtual.com/articulos/iedee/v12/123077.pdf.

43 REASON, James. The contribution of Latent Human Failures to the Breakdown of Complex Systems. Philosophical Transactions of the Royal Society Series B [Internet]. 1990 [citado 13 ago 2011]. 12 (327). p. 475-484. Disponible en: http://www.ncbi.nlm.nih.gov/ pubmed/1970893.

44 ORGANIZACIÓN INTERNACIONAL DE TRABAJO. Directrices relativas a los sistemas de gestión de la seguridad y la salud en el trabajo ILO - OHS. 2001. 43 p.

45 FERNÁNDEZ, Beatriz Op cit.
- Una política en la que la alta dirección fije su compromiso y visión para la organización.

- Una planificación para la identificación de peligros/riesgos, evaluación, control y respuesta a la emergencia donde se identifiquen los estándares aplicables (legales, por ejemplo), y se fijen los objetivos a mediano/largo plazo, así como las acciones para conseguirlos.

- Una estructura organizacional que refleje las responsabilidades en SST.

- Involucrar a los representantes de los trabajadores. Es un recurso imprescindible.

- Comunicación: existencia de unas vías de comunicación bidireccionales entre la Dirección y los trabajadores.

- Ejecutar y operar los planes y procesos de gestión. Tener presente su relación con actividades como la valoración de riesgos o las auditorías. Representa la práctica del sistema.

- Medida de mejoras a través de estadísticas laborales 0 auditorías que evalúen el sistema en su conjunto.

- Acciones preventivas y correctivas. Aproximación sistemática con el fin de identificar oportunidades para prevenir accidentes e incidentes.

- Revisión del sistema por la Dirección. Evaluación de lo apropiado del sistema de gestión para la organización, teniendo en cuenta las mejoras conseguidas.

- Progreso continuo. Es imprescindible un compromiso para gestionar de forma proactiva la seguridad y la salud en el trabajo.

Por otro lado, la National Occupational Health \& Safety Commission de Australia determina que la Teoría General de Sistemas sugiere la existencia de cuatro requisitos 
para los sistemas de gestión, específicamente los relacionados con los riesgos laborales, que son ${ }^{46}$.

- Los objetivos del sistema (éticos, económicos, legales y de tipo empresarial, no obstante, no todos los sistemas necesitan tener los mismos objetivos).

- Las especificaciones de los elementos del sistema y sus interrelaciones.

- La identificación de las relaciones entre este sistema de gestión y otros en la organización.

- Los requerimientos para el mantenimiento del sistema.

Adicionalmente, la International Occupational Hygiene Association (Asociación Internacional de Higiene Ocupacional) caracteriza a los sistemas de gestión de prevención de riesgos laborales, mediante cuatro elementos genéricos esenciales: las entradas, los procesos, las salidas o resultados y la retroalimentación ${ }^{47}$. Esto se relaciona con el enfoque basado en procesos descrito por la ISO ${ }^{48}$, el cual introduce la gestión horizontal, y conlleva la unificación de diferentes perspectivas hacia las metas principales de la organización.

Por otra parte, la OHSAS (Occupational Health and Safety Assessment Series) corresponde a una serie de normas de evaluación en materia de seguridad industrial y salud ocupacional, la cual consta de seis dimensiones: política seguridad y salud ocupacional, planificación, implementación y operación, verificación y acción correctiva, revisión por parte de la gerencia y mejora

46 RUBIO, Juan Carlos. Op cit. P. 57

47 Ibíd.

48 ISO. Orientación sobre el concepto y uso del "Enfoque basado en procesos" para los sistemas de gestión. ISO/TC 176/SC 2/N 544R2; 2003. Disponible en: http://www.icontec.org/BancoMedios/ Documentos\%20PDF/procesos.pdf. continua ${ }^{49}$. Este modelo es más flexible que la norma UNE 81900:1996, debido a que requiere ser compatible con otros sistemas, además, es aplicable a las pequeñas y medianas empresas. Sumado a ello, la OHSAS es más clara en la responsabilidad de la dirección y los recursos para el desarrollo de las actividades preventivas en la empresa, y para ello sugiere mecanismos para retroalimentar la gestión a partir de las actividades de preparación y respuesta a las mismas $5^{50}$. Uno de los cambios más importantes entre la versión de la OHSAS de 1999 y la del 2007 es que en esta última se da un mayor énfasis e importancia a la dimensión de la salud.

En Colombia, a partir de la Ley 1562 de 201251, se hizo obligatorio para todo tipo de organizaciones crear un sistema de gestión en seguridad y salud en el trabajo (SG-SST). Este aspecto fue reglamentado dos años más tarde a través del Decreto 1443 de 201452, en el cual se aborda el SG-SST desde las Directrices relativas a los sistemas de gestión de la seguridad y la salud en el trabajo de la OIT.

Otro de los avances logrados hasta el momento es la creación de una nueva norma certificable liderada por

49 RODRÍGUEZ, Yuber Liliana. Estrategias para el mejoramiento de la gestión de la salud y seguridad en el trabajo frente a las formas de vinculación en plantas de un grupo empresarial del sector industrial de Bogotá D.C. Tesis de maestría en Salud y Seguridad en el Trabajo - perfil investigativo. Bogotá. D.C: Universidad Nacional de Colombia. Facultad de Enfermería. 2012.

50 RUBIO, Juan Carlos y BENAVIDES, Carlos. Nuevas perspectivas en la normalización de la gestión de la seguridad y salud en el trabajo. 2011 [citado 16 nov 2011]. Disponible en: http://io.us.es/ cio2001/cio-2001/cd/Art\%C3\%ADculos/UMA/UMA-3.pdf

51 COLOMBIA. CONGRESO DE LA REPÚBLICA. Ley 1562 (11, julio, 2012). Por la cual se modifica el sistema de riesgos laborales y se dictan otras disposiciones en materia de salud ocupacional. Bogotá D.C.: Presidencia de la República.

52 COLOMBia. CONGRESO DE LA REPÚBliCA. Decreto 1443 (31, julio, 2014). Por el cual se dictan disposiciones para la implementación del sistema de gestión de la seguridad y salud en el trabajo (SG-SST). Bogotá D.C.: Ministerio de Trabajo. 
la ISO, la cual se denomina ISO 45001. Esta propuesta surge con el ánimo de alinear esta norma con otras, tales como la ISO 9001 y la ISO 14001, por cuanto se espera que esta modificación favorezca la integración de los sistemas de gestión en las organizaciones. No obstante, en la presente investigación no se profundiza en esta modificación normativa puesto que no hace parte del alcance del presente artículo.

En general, los modelos estandarizados de gestión en su mayoría se basan en el ciclo de Deming o PHVA, el cual tiene cuatro fases: planeación, elaboración o ejecución, verificación y, finalmente, ajuste. Si bien es cierto que los modelos descritos anteriormente muestran sus propias particularidades, el empleo es el elemento común de dicho ciclo. Barral reconoce que para la gestión de los servicios de la salud, se tienen en cuenta criterios que provienen de las ciencias "duras", como los que se observan en la planificación operativa y científica, y otros que son propios de las ciencias sociales, como los utilizados para la gestión del personal o para estudios económico-administrativos ${ }^{53}$.

En el caso de la SST, "planificar" es establecer una política de SST, es elaborar planes que incluyan la asignación de recursos, la facilitación de competencias profesionales, la organización del sistema, la identificación de los peligros y la evaluación de los riesgos. La fase "hacer" se refiere a

53 BARRAL, María. La gestión integrada y la dinámica de sistemas: criterios a aplicar en los laboratorios clínicos. Red Acta Bioquímica Clínica Latinoamericana [Internet]. 2007 [citado 13 ago 2011]; 41 (3). Disponible en: http://www.scielo.org.ar/scielo. php?pid=S0325-29572007000300016\&script=sci_arttext. la aplicación y puesta en práctica del programa de SST. La fase "verificar" se centra en evaluar los resultados tanto activos como reactivos del programa. Por último, la fase "actuar" cierra el ciclo con un examen del sistema en el contexto de la mejora continua y la preparación del sistema para el próximo ciclo $0^{54}$.

Otros autores, desde una perspectiva diferente, señalan que los elementos necesarios para la GSST se pueden sintetizar en cuatro categorías o factores: el compromiso de la dirección, la gestión de riesgos, la formación y comunicación, y la participación de los trabajadores. Así mismo, describen tres categorías de resultados que corresponden a los resultados en las personas, en la sociedad y en la organización ${ }^{55}$.

\section{Las implicaciones de flexibilización laboral en la GSST del sector salud}

Finalmente, la flexibilización laboral ha traído consigo una serie de exigencias tanto para los trabajadores como para las organizaciones del sector de la salud, que se ilustran en el Cuadro 2, las cuales han transformado el proceso salud- enfermedad en las poblaciones trabajadoras, y a su vez han llevado a los individuos a incrementar su nivel de formación y de conocimiento para poder convertirse en trabajadores polifuncionales.

\section{ORGANIZACIÓN INTERNACIONAL DEL TRABAJO. Op cit.}

55 GARCÍA, Susana, MANZANEDO, Miguel MARISCAL, Miguel,y ESPINOSA, Juan Factores que determinan el éxito de la gestión de la salud y seguridad. Escuela Politécnica Superior, Burgos. [citado 13 ago 2011]. Disponible en: http://io.us.es/cio2001/Cio-2001/cd/ Materia/Materia_11.htm. 
Cuadro 2. Exigencias de la flexibilización laboral

\begin{tabular}{|c|l|}
\hline \multicolumn{1}{|c|}{ ACTORES } & \multicolumn{1}{c|}{ EXIGENCIAS } \\
\hline \multirow{4}{*}{ TRABAJADOR } & $\begin{array}{l}\text { Fortalecimiento de valores } \\
\text { - Responsabilidad } \\
\text { - Compromiso con su quehacer y su proyecto } \\
\text { - Sentido de pertenencia } \\
\text { Capacidad de adaptación y de cambio } \\
\text { - Variedad de habilidades y capacidades }\end{array}$ \\
\cline { 2 - 3 } & Rotación interna del personal, para lo cual se requiere incrementar la capacitación del mismo. \\
\cline { 2 - 3 } ORGANIZACIÓN & Fraǵmentación del ciclo productivo - Inteǵración horizontal \\
\cline { 2 - 3 } & Articulación en relaciones interempresariales \\
\cline { 2 - 3 } & Propiciar la gestión que favorezca la autonomia funcional \\
\hline
\end{tabular}

Fuente: elaboración propia (basada en Rincón, María y Rodríguez, Isabel 2005; Torres, Sergio, y Angel, Víctor Manuel, 2004; Echeverría, Magdalena, 2003.

Dentro de las exigencias de la flexibilización laboral se encuentra la autonomía del trabajador. Este es un elemento protector de la salud de los trabajadores ${ }^{56}$ si se consideran los múltiples cambios que viven las organizaciones del sector de la salud en la actualidad como producto de la incursión de nuevas tecnologías y procesos de trabajo para responder a las demandas sociotécnicas. Sin embargo, en muchos casos los recursos son limitados, lo cual genera que el trabajador se exponga a nuevos factores de riesgo que afectan su salud y su calidad de vida.

Por otra parte, el fortalecimiento de valores se refleja en las características básicas del trabajo, es decir, en la variedad de habilidades, la identificación de la tarea, el significado de la tarea, la autonomía y la retroalimentación en el trabajo, al permitirle al trabajador de la salud comprender el significado de la experiencia, la responsabilidad experimentada por el proceso desarrollado y el conocimiento de los resultados de las actividades realizadas como producto de su trabajo. Lo anterior está asociado con la motivación interna del trabajador

56 CHUNG-YAN, Greg The Nonlinear Effects of Job Complexity and Autonomy on Job Satisfaction, Turnover, and Psychological WellBeing. Journal of Occupational Health Psychology [Internet]. 2010 [citado 13 ago 2011]; 15 (3): 237-251. Disponible en: http://www. ncbi.nlm.nih.gov/pubmed/20604631. de la salud, la cual se ve reflejada en el compromiso organizacional, y por ende, en la participación y en el desempeño laboral, así como en la mitigación/desapariación de síntomas físicos y psicosomáticos ${ }^{57}$.

En cuanto a la capacidad de adaptación y de cambio, el trabajador se ve obligado a responder a las nuevas formas sociales y técnicas de división del trabajo. Para ello necesita formación (educación), la cual le permitirá desarrollar las habilidades para afrontar las tareas en cambio constante de los procesos de producción y prestación de servicios de salud ${ }^{58}$. A su vez, la formación del personal podrá favorecer un rol más creativo e innovador dentro de la organización.

A partir de dichos cambios y exigencias generados por la flexibilización laboral, las organizaciones de diversos sectores intentan crear nuevos modelos para gestionar la salud y la seguridad en el trabajo, tal como se muestra en el Cuadro 3.

57 HUMPHREY, Stephen, NAHRGANG, Jennifer y MORGESON, Frank. Integrating motivational, social, and contextual work design features: A meta-analytic summary and theoretical extension of the work design literature. Journal of Applied Psychology [Internet]. 2007 [citado 13 ago 2011]. 92 (5). p. 1332-1356. Disponible en: http://www.ncbi.nlm.nih.gov/pubmed/17845089.

58 ECHEVERRÍA, (falta nombre). Op cit. 
Cuadro 3. Transformación que requiere la GSST

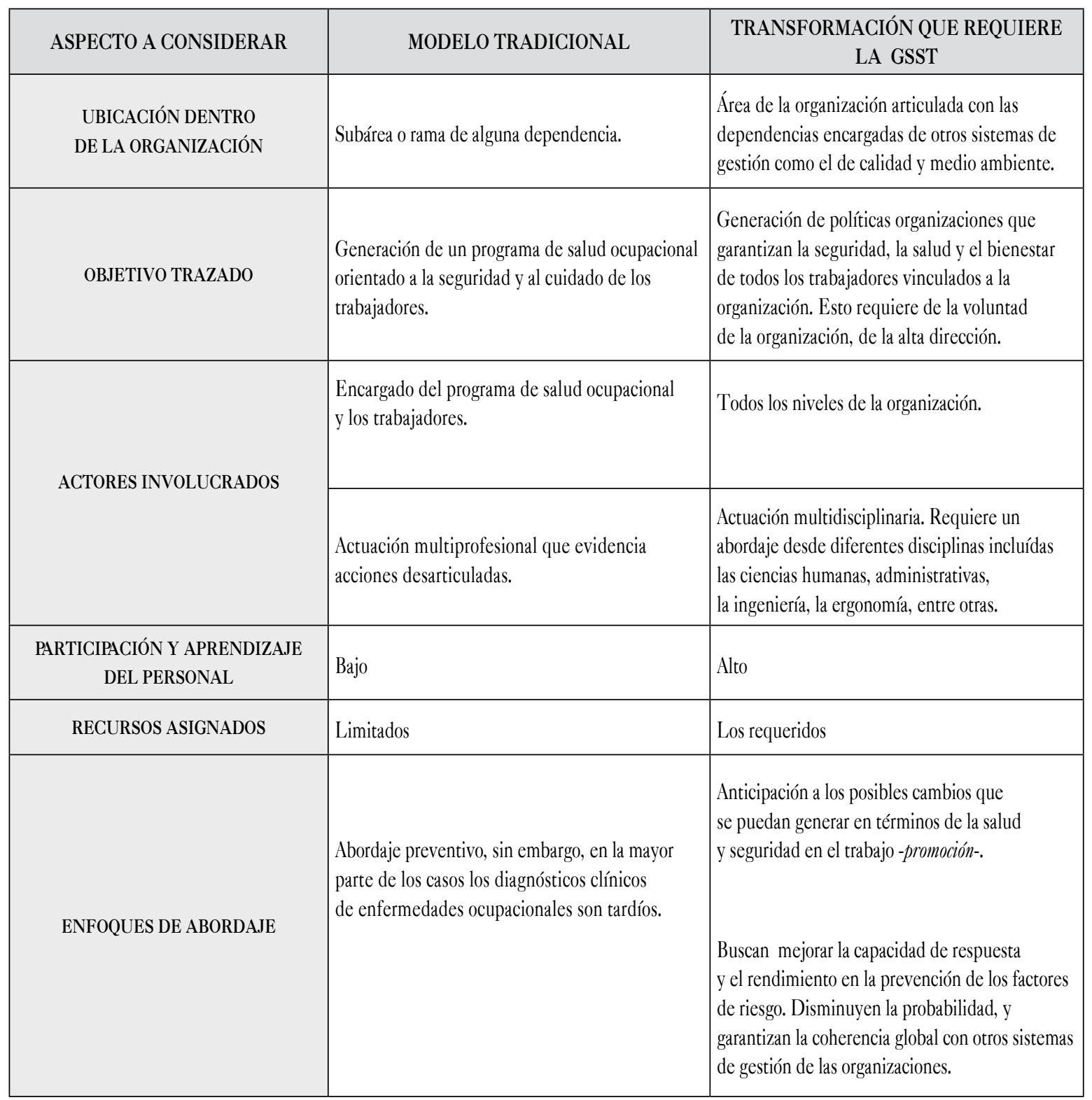

Fuente: elaboración propia, basada en Drais, Eric y Favaro, Marc. 2005.

Algunos autores consideran que los sistemas de GSST son enfoques integrales de prevención y gestión de los riesgos laborales, los cuales se fundamentan en una línea de base que siguen un proceso de cambio dirigido y apoyado por la organización respectiva ${ }^{59}$. Esta

59 DRAIS, Eric y FAVARO, Marc. Étude menée par le département Homme au travail [Internet]. 2006 [citado 13 ago 2011]. Disponible en: http://www.inrs.fr/htm/observation_evaluation_usages_systemes_management.html. necesidad de cambio en la GSST surge como respuesta a las transformaciones que ha generado la flexibilización laboral en las organizaciones del sector de la salud, las cuales implican tener en cuenta al trabajador y su puesto de trabajo, así como comprender aspectos relacionados con la dirección. como la creación de una política institucional acorde con los elementos misionales y el cuidado de la salud y seguridad de la población trabajadora del sector salud. 
Como bien se señaló en apartados anteriores, las dinámicas organizacionales presentan una mezcla de modelos tradicionales y actuales. Por ello se deben involucrar componentes de la gestión que cobijen a toda la población trabajadora de las organizaciones y que respondan a las necesidades reales de los trabajadores del sector de la salud.

En general, la adecuada GSST tiene implicaciones estratégicas para la organización dado que permite reducir pérdidas humanas y costos financieros directos e indirectos, y por ende, aumenta los beneficios para la misma. Adicionalmente, genera un efecto positivo sobre la motivación y satisfacción de los trabajadores, lo que incide sobre el clima organizacional al ser percibida como garante de los intereses sociales de los trabajadores. Esto a su vez redunda en el aumento de la productividad y creatividad de los trabajadores, y en la identificación de los trabajadores con los objetivos misionales de la organización ${ }^{60}$.

\section{CONCLUSIONES}

La flexibilización laboral es producto de la globalización. Esta ha influenciado todos los sectores, no obstante, uno de los más afectados ha sido el sector de la salud. Esta realidad se ha evidenciado en la inestabilidad laboral de los trabajadores, causada por el cambio en las modalidades de contratación, la susceptibilidad de despido y de rotación dentro de la organización; en el descentramiento del trabajo; la segmentación de la mano de obra y la reducción de la libertad sindical. Todo lo anterior genera el abaratamiento del costo de la mano de obra, y por ende, la reducción del salario real. Este hecho refuerza la flexibilización de los horarios en función de las necesidades productivas.

La GSST es un proceso sistemático y organizado, cuyo fin es la protección de la salud y el fomento de la seguridad

60 FERNÁNDEZ, MONTES y VÁZQUEZ. Op cit. de la población laboral en una organización del sector salud. La GSST permitirá la incorporación organizada de los componentes de la salud y seguridad en el trabajo, para una verdadera gestión de los mismos, que lleve a complementar la gestión de las organizaciones en aspectos que no han sido tradicionalmente tenidos en cuenta en un nivel suficiente para lograr beneficios organizacionales y para las personas.

Por otra parte, cualquier acción que se emprenda en pro de la GSST debe estar alineada con los objetivos que establece cada organización, de modo que se garantice la apropiación tanto de la dirección como del grupo de trabajadores.

El enfoque de los sistemas de GSST se articula con otros sistemas de gestión, además, permite la retroalimentación de cada una de las actividades para mejorar continuamente sus procesos. Lo anterior permite responder a las necesidades de los trabajadores y a modificar actividades para mejorar la permanencia en el mercado y competir dentro del mismo.

\section{RECOMENDACIONES}

El campo de la GSST en Colombia sigue siendo un tema poco explorado. Se recomienda estudiar su conceptualización y bases epistemológicas en mayor profundidad. Estos aspectos son esenciales para una posterior identificación de estrategias y herramientas que permitan su adecuada implantación en las organizaciones del sector de la salud.

Por otra parte, aunque la flexibilización laboral es un tema que ha sido analizado por diversos autores, este requiere estudiarse con más especificidad en lo concerniente a la identificación de mecanismos para la mitigación de sus efectos en la salud y seguridad de los profesionales en salud. 


\section{REFERENCIAS}

AÑEZ, Carmen. Estrategias de contratación de recurso humano en las empresas de servicios. Fermentum [Internet]. 2007 [citado 13 ago 2011]. 48. p. 123-141. Disponible en internet: http://www2.scielo.org.ve/ pdf/ferm/v17n48/art07.pdf.

ARENAS, Eraclio, PIEDRAHITA, Camilo y PLATA, Juan Miguel. Flexibilización y derecho laboral. Opinión Jurídica [Internet]. 2007 [Citado 13 ago 2011]. 5 (9). p. 163-171. Disponible en: http://redalyc.uaemex.mx/pdf/945/94550909.pdf.

ARENAS, Eraclio, PIEDRAHITA, Camilo y PLATA, Juan Miguel. Marco jurídico de las cooperativas de trabajo asociado. Opinión Jurídica [Internet]. 2007 [citado 13 ago 2011]. 6 (11). p. 34-45. Disponible en: http://redalyc.uaemex.mx/src/inicio/ ArtPdfRed.jsp?iCve=94501103.

BARRAL, María. La gestión integrada y la dinámica de sistemas: criterios a aplicar en los laboratorios clínicos. Red Acta Bioquímica Clínica Latinoamericana [Internet]. 2007 [citado 13 ago 2011]. 41 (3). Disponible en: http://www.scielo.org.ar/scielo.php?pid $=$ =S0325-29572007000300016\&script=sci_arttext.

BETANCOURT, Oscar. Enfoque alternativo de la salud y seguridad en el trabajo. En: IESS. Prevención es desarrollo. vol.1. 2007. p. 3-16.

CHUNG-YAN, Greeg. The Nonlinear Effects of Job Complexity and Autonomy on Job Satisfaction, Turnover, and Psychological Well-Being. Journal of Occupational Health Psychology [Internet]. 2010 [citado 13 ago 2011]. 15 (3). p. 237-251. Disponible en: http://www.ncbi.nlm.nih.gov/pubmed/20604631.

CODY, Marisue. Vertical integration strategies: Revenue effects in hospital and medicare markets. Hospital \& Health Services Administration. University of
Arkansas for Medical Sciences, College of Nursing [Internet]. 1996 [Citado 13 ago 2011]. 41 (3). p. 343-357. Disponible en: http://www.ncbi.nlm.nih. gov/pubmed?term $=$ Vertical $\% 20$ integration $\% 20$ strategies\%3A\%20revenue\%20effects\%20in\%20 hospital\%20and\%20Medicare\%20markets.

COLLER, Xavier La empresa flexible: Estudio sociológico del impacto de la flexibilidad en el proceso de trabajo. Centro de Investigaciones Sociológicos. Madrid, España. 1997. 100 p.

COLOMBIA. CONGRESO DE LA REPÚBLICA. Decreto 1443 (31, julio, 2014). Por el cual se dictan disposiciones para la implementación del sistema de gestión de la seguridad y salud en el trabajo (SG-SST). Bogotá D.C.: Ministerio de Trabajo.

COLOMBIA. CONGRESO DE LA REPÚBLICA. Ley 1562 (11, julio, 2012). Por la cual se modifica el sistema de riesgos laborales y se dictan otras disposiciones en materia de salud ocupacional. Bogotá D.C.: Presidencia de la República.

COLOMBIA. CONGRESO DE LA REPÚBLICA. Ley 789 (27, diciembre, 2002). Por la cual se dictan normas para apoyar el empleo y ampliar la protección social y se modifican algunos artículos del Código Sustantivo de Trabajo.

COLOMBIA. CONGRESO DE LA REPÚBLICA. Ley 50 (28, diciembre, 1990). Por la cual se introducen reformas al Código Sustantivo del Trabajo y se dictan otras disposiciones. Bogotá D.C.: Alcaldía de Bogotá.

CORIAT, Benjamín. Globalización de la economía y dimensiones macroeconómicas de la competitividad. CONICIT y el CREEDLA del CNRS.1994.

DEPESEX/BCN/Serie Estudios. La flexibilidad laboral en los países de economías avanzadas y de 
América Latina. El caso chileno. Biblioteca del Congreso Nacional de Chile [Internet]. 2005 [citado 13 ago 2011]. 318. Disponible en: http:// www.ben.cl/bibliodigital/pben/estudios/2005/estudios_pdf_estudios/nro318.pdf.

DRAIS, Eric y FAVARO, Marc. Étude menée par le département Homme au travail [Internet]. 2006 [citado 13 ago 2011]. Disponible en: http://www. inrs.fr/htm/observation_evaluation_usages_systemes_management.html.

ECHEVERRÍA, Magdalena. Aportes para el debate sobre flexibilidad laboral. Dirección del Trabajo. Santiago de Chile. Colección IDEAS. 2003.

ERMIDA, Oscar. Globalización y relaciones laborales. En: Revista Venezolana de Gerencia [Internet]. 1999 [citado 13 ago 2011]. 4 (9). Disponible en: http://www.cinterfor.org.uy/public/spanish/ region/ampro/cinterfor/publ/sala/ermida/globaliz/ globaliz.pdf.

FARNÉ, Stefano. Las cooperativas de trabajo asociado en Colombia: Balance de la política gubernamental, 2002-2007. Economía Institucional [Internet]. 2008 [citado 13 ago 2011]. 10 (18). p. 261-285. Disponible en: http://www.economiainstitucional. com/pdf/No18/sfarne18.pdf.

FERNÁNDEZ, Beatriz, MONTES, José Manuel y VÁZQUEZ, Camilo. Desarrollo y validación de una escala de medición para el sistema de gestión de la seguridad laboral. Investigaciones Europeas de Dirección y Economía de la Empresa [Internet]. 2006 [citado 13 ago 2011]. 12 (3). p. 77-93. Disponible en: http://www.aedem-virtual.com/ articulos/iedee/v12/123077.pdf.

FERNÁNDEZ, Ricardo. Los distintos sistemas de gestión de la salud y seguridad en el trabajo. Gestión Práctica de Riesgos Laborales. Editorial Club Universitario. San Vicente. España. 2007. 375 p. I
GARCÍA, SUSANA, MANZANEDO, Miguel, MARISCAL, Miguel y ESPINOSA, Juan. Factores que determinan el éxito de la gestión de la salud y seguridad. Escuela Politécnica Superior, Burgos. [citado 13 ago 2011]. Disponible en: http://io.us.es/ cio2001/Cio-2001/cd/Materia/Materia_11.htm.

HUMPHREY, Stephen, NAHRGANG, Jennifer y MORGESON, Frank. Integrating motivational, social, and contextual work design features: A metaanalytic summary and theoretical extension of the work design literature. En: Journal of Applied Psychology [Internet]. 2007 [citado 13 ago 2011]. 92 (5). p. 1332-1356. Disponible en: http://www. ncbi.nlm.nih.gov/pubmed/17845089.

ISO. Orientación sobre el concepto y uso del "Enfoque basado en procesos" para los sistemas de gestión. ISO/TC 176/SC 2/N 544R2; 2003. Disponible en: http://www.icontec.org/BancoMedios/Documentos\%20PDF/procesos.pdf.

KLAU, Friedrich y MITTELSTADT, Axe. Labour market flexibility. OECD [Internet]. Disponible en internet: http://www.oecd.org/dataoecd/ 55/25/35558438.pdf.

UNIVERSIDAD NACIONAL DE COLOMBIA. Línea de investigación en gestión, organización y regulación de la salud y seguridad en el trabajo. Actas de reunión; 2010.

LIZAMA, Claudio. Flexibilidad laboral en la Unión Europea y Estados Unidos. En: Revista de la Escuela de Derecho de la Universidad de Chile. 2003. p. 35-51.

LUCENA, Héctor. Relaciones de Trabajo en el Nuevo Siglo. Caracas Fondo editorial Tropykos. 2004. 300 p.

MILANO, Elías. Del Fordismo a la flexibilidad laboral: supuestos, crisis, y realidades de la regulación social. Caracas. Biblioteca Omegalfa.1997. 55 p. 
MOTA, Ana Elizabete. Cultura da Crise e Seguridade Social: Um Estudo Sobre as Tendências da Previdência e da Assistência Social Brasileira nos Anos 80 e 90. São Paulo: Cortez, 1995. 248 p.

ISO. Norma Internacional ISO 9000:2005. Sistema de gestión de la calidad. Fundamentos y Vocabulario. Ginebra.

OBSERVATORIO DE RECURSOS HUMANOS EN SALUD. Relaciones laborales en el sector salud; el Sistema General de Seguridad Social en Salud de Colombia: El caso de la ciudad de Cali. Bogotá. Ministerio de la Protección Social y Organización Panamericana de la Salud. 2006.

OCHOA, Haydée y GAMBOA, Teresa. Hacia la Gerencia Pública en Venezuela: el caso de la administración tributaria. En: Revista Centroamericana de Administración Pública. No 37. 1999. p. 93-116.

ORGANIZACIÓN INTERNACIONAL DE TRABAJO. Directrices relativas a los sistemas de gestión de la seguridad y la salud en el trabajo ILO - OHS. 2001.

ORGANIZACIÓN INTERNACIONAL DEL TRABAJO. Sistema de gestión de la SST: una herramienta para la mejora continua. 1a. ed. 2011.

REASON, James. The contribution of Latent Human Failures to the Breakdown of Complex Systems. Philosophical Transactions of the Royal Society Series B [Internet]. 1990 [citado 13 ago 2011]. No. 12 (327). p. 475-484. Disponible en: http://www. ncbi.nlm.nih.gov/pubmed/1970893.

RINCÓN, María y RODRÍGUEZ, Isabel. Flexibilización Laboral en el sector salud: Una estrategia en la Gobernación del Zulia. Gaceta Laboral [Internet]. 2005 [citado 13 ago 2011]. 11 (3). Disponible en: http://www.scielo.org.ve/scielo.php?pid=S131585972005000300003\&script=sci_arttext.
RODRÍGUEZ, Yuber Liliana y MOLANO, Jorge. Gestión de la seguridad y salud en el trabajo frente a las formas de vinculación en un consorcio empresarial. Universidad Nacional de Colombia. En: La investigación en Salud y Seguridad en el trabajo Realidades y desafíos actuales. Bogotá. D.C: Universidad Nacional de Colombia. 2014. 189 p.

RODRÍGUEZ, Yuber Liliana y MOLANO, Jorge . Adaptación de una herramienta para la evaluación de la gestión de la salud y seguridad en el trabajo. En: El Hombre y la Máquina. 2012. vol. 40. p. 7-21.

RUBIO, Juan Carlos. Gestión de la prevención de riesgos laborales: OHSAS 18001 - Directrices OIT para su integración con calidad y medioambiente. España: Ediciones Díaz de Santos. 2006. Falta número de páginas.

RUBIO, Juan Carlos y BENAVIDES, Carlos. Nuevas perspectivas en la normalización de la gestión de la seguridad y salud en el trabajo. 2011 [citado 16 nov 2011]. Disponible en: http://io.us.es/cio2001/ cio-2001/cd/Art\%C3\%ADculos/UMA/UMA-3.pdf.

TORRES, Sergio y ANGEL, Víctor Manuel. Outsourcing de servicios en la prestación de servicios de salud de Bogotá. Gerencia y Políticas de Salud [Internet]. 2004. [citado 13 ago 2011].3 (7). p. 101 - 113. Disponible en: http://redalyc.uaemex.mx/src/inicio/ArtPdfRed.jsp?iCve=54500707.

VALDÉS, Fernando. Descentralización productiva y desorganización del derecho del trabajo. Universidad Complutense de Madrid. [citado 13 nov 2011]. Disponible en: http://www.ruct.uva.es/pdf/Revista\%202/202.pdf 